\title{
Bruxismo e sua relação com o sistema nervoso central: Revisão de Literatura
}

\author{
Bruxism and its relationship with the central nervous system: Literature Review
}

Dayana Campanelli Morais

Especialista em Prótese Dentária pela UFRJ

Aleli Tôrres de Oliveira

Especialista em Dor Orofacial e DTM

Professora Substituta da FO/UFRJ

André Antonio Monteiro

MS

\section{Maria José Santos Alencar}

Professora Doutora do Departamento de Prótese e Materiais Dentários da FO/UFRJ

Coordenadora do Curso de Especialização em Prótese Dentária

\section{Resumo}

O bruxismo é descrito como um movimento orofacial, denominado parafunção, cuja etiologia é complexa e multifatorial. O presente estudo teve como objetivo, facilitar 0 entendimento das possíveis causas dessa desordem e sua relação com o Sistema Nervoso Central, através de uma revisão de literatura. Alguns autores $(4,23,19)$ afirmam a hipótese de 0 bruxismo ser mediado centralmente, tornando 0 estresse emocional e a ansiedade, fatores importantes para seu desencadeamento. Como auxílio diagnósti$\mathrm{CO}$, a polissonografia (PSG) é uma importante ferramenta, porém devido ao custo ainda oneroso, poucos estudos foram encontrados correlacionando a PSG e o bruxismo, sendo de grande importância que mais pesquisas sejam realizadas sobre 0 assunto.

Palavras-chave: bruxismo do sono; dopamina e bruxismo; polissonografia e bruxismo.

\section{Abstract}

Bruxism is described as an orofacial movement called parafunction, whose etiology is complex and multifactorial. This study aimed to facilitate the understanding of the possible causes of this disorder and its relation with the central nervous system, through a literature review. Some authors 44,23 , 19) affirm the hypothesis of bruxism be mediated centrally, making the emotional stress and anxiety important factors for its appearance. For diagnosis, polysomnography (PSG) is an important tool, however due it's onerous cost, few studies were found correlating the PSG and bruxism, which is a matter of great importance that more research be conducted on the subject.

Keywords: Sleep bruxism; dopamine and bruxism; polysomnography and bruxism.

\section{Introdução}

A atividade parafuncional é um dos fatores principais associados à DTM. As atividades da musculatura mastigatória podem ser divididas em dois tipos básicos: funcional que inclui mastigar, falar e deglutir e parafuncional (não funcional), que inclui apertar ou ranger os dentes (bruxismo) e vários outros hábitos orais. Os danos causados aos portadores de hábitos parafuncionais motivam estudos que buscam desenvolver terapias mais eficazes para controle e tratamento das mesmas, sendo o bruxismo a parafunção mais pesquisada (7).

O bruxismo é descrito como um distúrbio de movimentos estereotipados, geralmente associado com microdespertares. Existe também o bruxismo da vigília. Os sons produzidos pela fricção dos dentes normalmente são percebidos pelo companheiro. Entre os efeitos indesejáveis resultantes desse distúrbio estão: desgaste dos dentes, hipersensibilidade dentária a estímulos térmicos, dor orofacial e cefaleia temporal $(2,6)$.

A relação de substâncias neuroquímicas, chamadas catecolaminas, com o bruxismo, está em destaque atualmente, porém, ainda há poucos estudos descrevendo os detalhes desse assunto. A dopamina é uma dessas substâncias e corresponde a mais da metade do conteúdo de catecolaminas do SNC (23). Acredita-se que ela pode estar diretamente relacionada com a inibição de movimentos espontâneos e ter ligação com manifestações de estresse e ansiedade. Com isso, alterações de seus níveis podem ser responsáveis pelo desencadeamento de movimentos repetitivos coordenados, sugerindo o envolvimento do sistema dopaminérgico e da neurotransmissão central na etiologia do bruxismo $(22,21,19,3,24,25)$.

\section{Revisão de Literatura}

Segundo OKESON (15), estudos epidemiológicos revelam que a maior parte dos sintomas de DTM é relatada pela faixa etária de 20 a 40 anos e sua etiologia é complexa e multifatorial. Acredita-se que $85 \%$ a $90 \%$ da população rangem os dentes em algum grau durante a vida e somente cerca de $5 \%$ desses pacientes, apresenta o bruxismo como condição clínica.

CUNALI et al. (6) afirmaram que, até o momento, as melhores ferramentas para o diagnóstico de bruxismo e DTM em pesquisa são, respectivamente, a polissonografia (PSG) e o índice Reseach Diagnostic Criteria (RDC/TMD). Como o diagnóstico clínico do bruxismo por meio da anamnese apresenta limitações, o ideal seria que todos os prováveis bruxômanos fossem submetidos ao exame de PSG. O autor considera o exame importante para confirmar o diagnóstico, descartando outros movimentos orofaciais realizados durante o sono, tais como deglutição, tosse, grunhir ou alternar abertura e fechamento da boca, que podem ser confundidos com o bruxismo. Mas infelizmente, a PSG ainda é onerosa, pois depende de laboratório de sono estruturado, técnico capacitado e deslocamento do paciente $(1,11)$.

LAVIGNE (11), estudando o padrão eletromiográfico dos movimentos mandibulares durante o sono, sugeriu denominar esses movimentos de AMMR (Atividade Muscular Mastigatória Rítmica). A AMMR ocorre durante o sono cerca de uma a duas vezes por hora em indivíduos saudáveis. $\mathrm{O}$ 
que diferenciou indivíduos saudáveis de indivíduos bruxômanos foi a frequência da AMMR, que passou para quatro a doze vezes por hora de sono, e a amplitude eletromiográfica, entre $30 \%$ a $50 \%$ maior em indivíduos com bruxismo, demonstrando uma maior intensidade da atividade muscular neste grupo.

LAVIGNE (11) ainda relata que os eventos de bruxismo ocorrem principalmente durante o sono leve (N1 e N2) e mais raramente no sono REM (menos de 10\%). Grande parte dos episódios está associada a movimentos corporais leves e há uma correlação com microdespertares. Os microdespertares são atividades naturais que ocorrem de 8-15 vezes por hora durante o sono, onde há um aumento da atividade autonômica simpática com o aumento da ativação cardíaca e cerebral precedendo o início da atividade nos músculos suprahioideos e mastigatórios na forma de movimentos rítmicos mandibulares. Esse envolvimento do sistema nervoso autônomo com os movimentos mandibulares sugere que o bruxismo seja mediado centralmente por vários neurotransmissores principalmente pelo sistema dopaminérgico.

Para GUYTON (8), a dopamina pode causar o aumento dos batimentos cardíacos, náuseas, aumento do tônus dos músculos supra-hioides e início da atividade muscular mastigatória rítmica do masseter e, consequentemente, o ranger de dentes $(17,16,13,8,5)$.

A primeira evidência que o bruxismo do sono poderia estar ligado a neuroquímica cerebral surgiu do estudo de WINOCUR et al. (26), no qual um paciente com diagnóstico de Parkinson foi tratado para bruxismo com 1-3,4-dihydroxyphenylalanine (l-DOPA), um precurso da catecolamina. $\mathrm{O}$ autor também relatou que o estado hiperdopaminérgico poderia ser induzido pelo metilenodioximetanfetamina (MDMA) ou ecstasy, cocaína e anfetaminas. O ecstasy é uma droga que provoca energia, autoconfiança e empatia. Entre seus efeitos colaterais, a manifestação do bruxismo é relatada por, aproximadamente, $70 \%$ de seus usuários e tem sido atribuída à interação da droga com o sistema serotoninérgico central.

Um estudo realizado em 1980, por CLARK et al. (4), testou a hipótese de relação entre bruxismo do sono e estresse. Para isso, níveis de catecolaminas na urina foram avaliados em 20 pacientes com bruxismo e 10 sem bruxismo. Os critérios de diagnóstico foram presença de desgaste dentário, relato de dor nos músculos da face e hábito de apertar e/ou ranger os dentes. Como recurso, a eletromiografia (EMG) foi utilizada para medir a atividade do músculo masseter. Os pacientes com bruxismo foram divididos em atividade muscular: leve (grupo 1) e pesada (grupo 2). O nível urinário de adrenalina no grupo sem bruxismo foi de $5,19 \mu \mathrm{g} / \mathrm{ml}$. No grupo 1 , o resultado foi de $6,4 \mu \mathrm{g} / \mathrm{ml}$ e no grupo 2, 10,36 $\mu \mathrm{g} / \mathrm{ml}$. Os níveis médios urinários evidenciados para noradrenalina foram de 19,9 $\mu \mathrm{g} / \mathrm{ml}$ para o grupo sem bruxismo; $28,2 \mu \mathrm{g} / \mathrm{ml}$ no grupo 1 e $39,1 \mu \mathrm{g} / \mathrm{ml}$ no grupo 2 . Verificou-se uma correlação positiva entre bruxismo e os níveis de catecolaminas urinárias e, segundo os autores, esse resultado suporta a teoria que aponta o estresse emocional como um dos fatores etiológicos do bruxismo.

Em 1999, VANDERAS et al. (25) investigaram a relação entre os níveis de catecolaminas encontradas na urina e o desenvolvimento do bruxismo em 314 crianças de escola pública, de ambos os sexos, com idade entre 6 e 8 anos. O diagnóstico foi realizado a partir de exame clínico e anamnese, utilizando critério diagnóstico para bruxismo a presença de facetas de desgaste em molar permanente. Toda urina durante um período de 24 horas foi coletada de cada participante e analisada por cromatografia líquida de alta performance, com o intuito de avaliar a quantidade de catecolaminas. Nenhuma restrição alimentar foi aplicada. Bruxismo foi diagnosticado em 129 dos 273 participantes que completaram a coleta de urina. A análise evidenciou que a adrenalina, noradrenalina e a dopamina têm influência significativa na probabilidade do desenvolvimento do bruxismo.

KATO et al. (9) afirmaram que a contribuição do estresse psicossocial na etiologia do bruxismo não pode ser negligenciada e, que o tratamento cognitivo e comportamental, incluindo alterações no estilo de vida, podem ser benéficos. Placas de mordida podem ser úteis para proteger os dentes e alguns medicamentos, tais como benzodiazepínicos e relaxantes musculares, podem ser prescritos por curto período de tempo, particularmente quando existe dor secundária.

SERAIDARIAN (21) realizou estudo em Taubaté (2006), com quarenta indivíduos, de ambos os gêneros, na faixa etária entre 18 a 50 anos, portadores de dentição completa fixa. Para diagnóstico do bruxismo, foi realizado um questionário e exame clínico oral. Os critérios de inclusão para pacientes com bruxismo foram a presença de desgastes nas bordas incisais e/ou oclusais dos dentes, com alteração da morfologia dentária, além de relatos de familiares sobre ruído decorrente de ranger de dentes. Não participaram da pesquisa indivíduos que faziam uso de descongestionante nasal, droga broncodilatadora e antihipertensiva arterial (metildopa), antidepressivos tricíclicos, benzodiazepínicos, L-Dopa, tetraciclina, cloropromazina e cloridrato de etilfedrina (hipertensor arterial). Os participantes coletaram o volume total de urina excretada em um período de 24 horas. Os valores médios dos níveis urinários das catecolaminas evidenciados pela cromatografia líquida de alta performance foram: em indivíduos do grupo controle a adrenalina obteve resultado $35,05 \mu \mathrm{g} / \mathrm{ml}$, a noradrenalina de $148,7 \mu \mathrm{g} /$ $\mathrm{ml}$ e a dopamina $201,7 \mu \mathrm{g} / \mathrm{ml}$; já nos bruxômanos os resultados foram de $111,4 \mu \mathrm{g} / \mathrm{ml}$ para a adrenalina, 261,59 $\mu \mathrm{g} /$ $\mathrm{ml}$ para a noradrenalina e $479,56 \mu \mathrm{g} / \mathrm{ml}$ para a dopamina. Com isso, concluiu-se que é grande a diferença dos níveis de catecolaminas encontrados nas urinas de pacientes com e sem bruxismo, evidenciando uma relação importante entre a quantidade desses neurotransmissores e a etiologia da referida parafunção.

NASCIMENTO et al. (14) realizaram um estudo transversal em 2009 com militares da ativa da Marinha da Guerra do Brasil, com o objetivo de verificar a presença de bruxismo 
e a sua relação com o estresse emocional. Foram selecionados 486 voluntários, independente de cor ou raça, com idade entre 19 e 48 anos. Destes, foram avaliados 259 (53,3\%) militares embarcados em navio (Organização Militar Operativa) e 227 (46,7\%) militares de Organização Militar de Terra (Administrativa). Os voluntários selecionados foram submetidos à avaliação psicológica, para obtenção do diagnóstico de estresse e, posteriormente, a um exame clínico. $\mathrm{O}$ critério de inclusão para paciente com bruxismo no exame clínico foi a presença de facetas de desgaste em esmalte e/ou dentina na face incisa e/ou oclusal dos dentes. Foram identificados dois grupos de pacientes: estressados e não estressados, sendo 103 pacientes com estresse (21\%) e 383 pacientes não estressados (79\%). Para correlacionar o estresse emocional ao bruxismo, a amostra contou com os 103 pacientes que apresentaram sintomatologia de estresse. Neste grupo procurou-se a presença ou ausência de evidências clínicas do bruxismo, onde 46 pacientes (44,7\%) apresentaram evidências clínicas de bruxismo e 57 pacientes $(55,3 \%)$ não apresentaram tais evidências. Encontrou-se uma predominância da associação dos militares do grupo administrativo com a presença de bruxismo, sendo este o grupo de militares de maior idade, acima de 30 anos.

\section{Discussão}

O sistema dopaminérgico parece ter um importante papel na etiologia do bruxismo do sono. Existem duas hipóteses relacionadas com as alterações dopaminérgicas: a hipótese hiperdopaminérgica demonstrada em estudos com pacientes portadores da doença de Parkinson e que apresentam ranger dos dentes durante tratamento com L-Dopa, além de estudos com o uso crônico de drogas antidopaminérgicas, causando hipersensibilidade dos receptores dopaminérgicos, podendo provocar manifestações de discinesia tardia e ranger de dentes. As anfetaminas, metilenodioximetanfetamina (MDMA) ou ecstasy e cocaína também poderiam induzir a um estado hiperdopaminérgico provocando ranger de dentes durante a vigília e o sono. A outra hipótese é a hipodopaminérgica que seria baseada em estudos que demonstram que a administração de doses baixas de L-Dopa, exercem efeito terapêutico, e atenuam episódios de ranger de dentes em casos de bruxismo do sono primário $(21,19,3,12,20)$.

Em pesquisas realizadas com o objetivo de quantificar as catecolaminas encontradas na urina, a possível relação desses neurotransmissores na etiologia do bruxismo foi confirmada. CLARCK et al. (4), VANDERAS (25) e SERAIDARIAN (21) verificaram a correlação positiva entre bruxismo do sono e estresse emocional.

Relação entre bruxismo e a síndrome das pernas inquietas (PLM) foi confirmada em cerca de $10 \%$ a $20 \%$ dos portadores de bruxismo do sono, com movimentos periódicos dos membros durante o sono. No tratamento de (PLM), é administrado doses baixas de L-Dopa e outros agonistas dopaminérgicos, resultando em redução da neurotransmis- são dopaminérgica, diminuindo os movimentos rítmicos das pernas de modo semelhante houve uma diminuição dos movimentos mandibulares nos indivíduos com bruxismo do sono, sugerindo assim acometimento pré-sináptico das células produtoras de dopamina $(21,20,10)$.

\section{Conclusão}

Pesquisas sobre as catecolaminas evidenciaram uma ligação importante entre a quantidade de neurotransmissores e o bruxismo, confirmando a hipótese do estresse emocional estar envolvido na etiologia da referida parafunção.

Podemos concluir que a etiologia do bruxismo ainda é obscura e vários são os fatores que podem estar relacionados com o seu desencadeamento. Alguns estudos relatam que certas medicações podem aumentar os eventos de bruxismo, embora a evidência ainda seja fraca, outros sugerem que possa haver uma predisposição genética.

Pesquisas sobre a parafunção com o exame PSG e os neurotransmissores centrais são necessárias, para que a eficácia no manejo dos pacientes possa ser melhorada. E o tratamento dos problemas clínicos associados ao bruxismo deve ser avaliado por uma equipe multidisciplinar capacitada para tal finalidade. 


\section{Referências ::}

1. ALOÉ, F, GONCALVES, LR, AZEVEDO, A, et al. Bruxismo durante o sono. Rev Neurociências 2003;11(1):4-17.

2. AMERICAN ACADEMY OF SLEEP MEDICINE. International classification of sleep disorders, revised: diagnostic and coding manual. Chicago, Illinois: American Academy of Sleep Medicine; 2001

3. ARESO, MP, GIRALT, MT, SAINZ, B, et al. Occlusal disharmonies modulate central catecholaminergic activity in the rat. J Dent Res 1999;78(6): 1204-13.

4. CLARK, GT, RUGH, JD, HANDELMAN, SL. Nocturnal masseter muscle activity and urinary catecholamine levels in bruxers. J Dent Res 1980; 59(10):1571-6.

5. CUCCIA, AM. Aetiology of sleep bruxism: a review of the literature. Recenti progress Med 2008; 99(6):322-8.

6. CUNALI, RS, BONOTTO, DMV, MACHADO, E, et al. Bruxismo do sono e disfunções temporomandibulares: revisão sistemática. Rev Dor 2012; 13(4):360-4.

7. DEKON, SFC, PELLIZZER, EP, ZAVANELLI, AC, et al. Reabilitação oral em paciente portador de parafunção severa. Rev Odonto Araçatuba 2003; 24(1):54-9.

8. GUYTON, AC, HALL, JE. Fisiologia humana e mecanismos das doenças. 6. ed. Rio de Janeiro: Guanabara Koogan, 1998. p. 325-82.

9. KATO, T, THIE, N, MONTPLAISIR, J, et al. Bruxism and orofacial movements during sleep. Dent Clin North Am 2001; 45:657-84.

10. LAVIGNE, GJ, MONTPLAISIR, J. Restless legs syndrome and sleep bruxism: prevalence and association among canadians. Sleep. 1994;17 (8):739-43.

11. LAVIGNE, GJ, ROMPRÉ, PH, MONTPLAISIR, JY. Sleep bruxism: validity of clinical research diagnostic criteria in a controlled polysomnographic study. J Dent Res 1996;75(1):546-52.

12. LOBBEZOO, F, NAEIJE, M. Bruxism is mainly regulated centrally, not peripherally. Journal of Oral Rehabilitation 2001; 28(12):1085-91.
13. MACEDO, CR. Bruxismo do sono. Rev Dental Press Ortodon Ortop Facial 2008; 13(2):18-22.

14. NASCIMENTO, AM, PIRES, AA, SANTOS, ACBM, et al. Associação do bruxismo ao estresse emocional: estudo transversal. RBO 2009;66(2):160-4.

15.OKESON, JP. Tratamento das Desordens Temporomandibulares e Oclusão. 7. Ed. Rio de Janeiro: Elsevier; 2013. p. 102-28.

16. PRIMO, PP, MIURA, CSN, BOLETA-CERANTO, DCF. Considerações fisiopatológias sobre bruxismo. Arq Ciênc Saúde UNIPAR 2009;13(3):263-6.

17. SERAIDARIAN, PI, CAVALCANTI, BN. A importância do bruxismo nas desordens temporomandibulares. In: GONÇALVES, EAN, GENTIL, SN. (Org.). Atualização Clínica em Odontologia. São Paulo: Artes Médicas; 2004. p. 303-20.

18. SERAIDARIAN, PI, JACOB, MF, SERAIDARIAN, P. Mecanismos neurológicos envolvidos na gênese do bruxismo. JBA. 2002; 2 (7): 240-6.

19. SERAIDARIAN, P. Níveis urinários de catecolaminas em indivíduos bruxômanos e não bruxômanos. Universidade de Taubaté, São Paulo, 2006.

20. SJOHOLM, TT, et al. The effect of propanolol on sleep bruxism hypothetical considerations based on a case study. Clin Auton Res 1996; 6(1): 37-40.

21. STRYER, L. Bioquímica. 3. ed. Rio de Janeiro: Guanabara Koogan; 1998. $881 \mathrm{p}$

22. SUNDÉN-KURONEN, B, et al. Influence of central dopaminergic and oral sensory stimulation on the tone of the rat masseter muscle. Acta Odontol Scand 1983; 41(3):159-66.

23. VANDERAS, AP, MENENAKOU, M, KOUIMTZIST, H, et al. Urinary catecholamine levels and bruxism in children. J Oral Rehabil 1999;26 (2):103-10.

24. WINOCUR, E, GAVISH, A, VOIKOVITCH, M et al. Drugs and bruxism: a critical review. Journal of Orofacial Pain. 2003; 17(2):99-111.

Recebido em: 23/07/2014 / Aprovado em: 26/08/2014

Dayana Campanelli Morais

Estrada dos Bandeirantes, 2698, casa 49

Rio de Janeiro/RJ, Brasil - CEP: 22775-110

E-mail: dayanacampanelli@gmail.com 